\title{
Pregnancy Screening Strategies for Potentially Challenging Patients Before Diagnostic Nuclear Medicine Procedures: Qualitative Survey Analysis
}

\author{
Daphne J. James ${ }^{1}$, Paul Cardew ${ }^{1,2}$, and Helen M. Warren-Forward ${ }^{1}$ \\ ${ }^{1}$ School of Health Sciences, University of Newcastle, Australia; and ${ }^{2}$ Hunter New England Imaging, Hunter New England Area \\ Health Service, Newcastle, Australia
}

Because of the ionizing radiation used in diagnostic nuclear medicine procedures, it is recommended that all female patients of childbearing age be questioned about their pregnancy status before the procedure begins. Several patient groups have been identified as potentially difficult to question: teenagers, unconscious or sedated patients, patients with language or cultural barriers, and patients with mental disability. Our aim was to capture the thoughts and opinions of nuclear medicine personnel in Australia and New Zealand regarding pregnancy screening strategies before diagnostic imaging procedures. Methods: Members of the Australian and New Zealand Society of Nuclear Medicine were invited to complete an online survey. Section 4 consisted of open-response questions asking participants to describe the strategies they use to question a patient about pregnancy status in 4 potentially difficult clinical scenarios. The content of the responses was analyzed. Results: For each question, 232 responses were recorded. The most commonly used strategies included questioning teenage girls away from their parents, referring to medical notes for unconscious patients, using an interpreter and visual aids for patients with language barriers, and asking a caregiver or relative of mentally disabled patients. Pregnancy testing was used when there was doubt about the patient's pregnancy status. Personal questions about menstrual and sexual history were often asked to determine the risk of pregnancy. Conclusion: The study revealed that a variety of strategies are used by nuclear medicine personnel in Australia and New Zealand to determine the pregnancy status of patients. A standardized practice guideline may be useful to ensure a consistent approach to questioning that would optimize the accuracy of pregnancy assessment and reduce the possibility of fetal irradiation.

Key Words: ionizing radiation; pregnancy; qualitative

J Nucl Med Technol 2013; 41:292-298

DOI: 10.2967/jnmt.113.125856

Received May 5, 2013; revision accepted Aug. 8, 2013.

For correspondence or reprints contact: Daphne James, School of Health Sciences, University of Newcastle, University Drive, Callaghan. N.S.W. 2308 Australia.

E-mail: daphne.james@newcastle.edu.au

Published online Oct. 21, 2013.

COPYRIGHT (C) 2013 by the Society of Nuclear Medicine and Molecular Imaging, Inc.
$\mathbf{T}$ here have been several articles published discussing the difficulties associated with determining pregnancy status before diagnostic imaging procedures using ionizing radiation (1-4). Nuclear medicine imaging procedures using ${ }^{99} \mathrm{~m} \mathrm{Tc}$ as the radionuclide typically give an effective dose of less than $10 \mathrm{mSv}$ for adults (5). However, doses of this size still pose a potential risk of biologic damage to a fetus if irradiated during maternal imaging procedures (6). Several international and national documents, such as publication 84 of the International Commission on Radiological Protection (6), report 128 of the National Council on Radiation Protection and Measurements (7), and publication 14.2 of the Australian Radiation Protection and $\mathrm{Nu}-$ clear Safety Agency (8), provide information on protection of the fetus from radiation. These documents discuss the potential risks to a fetus from maternal medical imaging using ionizing radiation. They recommend that all women of childbearing age be questioned about their pregnancy status before the procedure begins, that the reason for asking be explained to the patient to avoid offense and illicit a more truthful answer, and that multilingual signs be placed around medical imaging departments asking patients to notify staff if they think they might be pregnant.

There are, however, no clearly defined guidelines to help nuclear medicine personnel determine which patients to question about pregnancy status or the best approach to questioning them. Teenagers and patients with language barriers are among several groups that have been identified as being potentially difficult to question and gain a truthful and accurate response $(2,4)$.

A survey designed to investigate current practice and capture opinions about pregnancy determination in nuclear medicine in Australia and New Zealand was distributed to members of the Australian and New Zealand Society of Nuclear Medicine. The survey aimed to verify the findings from an earlier interview study (2), which had a limited number of participants. The design of the questionnaire and the results from sections 1-3 of the survey regarding demographic data, knowledge of regulations and policy, and current practice have been previously discussed in detail (7). The results revealed a lack of awareness of radiation protection guidelines 
for the fetus and confirmed wide variations in current practice for questioning patients about their pregnancy status. Participants indicated that they used various methods of questioning, including verbal questioning, verbal questioning with the patient signing a form, and the use of written questionnaires. The age for questioning ranged from 10 to $60 \mathrm{y}$, with $12 \mathrm{y}$ and $55 \mathrm{y}$ being the most common minimum and maximum ages of patients to be questioned.

This paper concentrates on the content analysis of the openresponse questions in section 4 of the survey, which asked participants to briefly describe how they would question the patient in 4 clinical scenarios: the young teenage patient, the unconscious or heavily sedated patient, the patient with language or cultural barriers, and the patient with mental disability.

\section{MATERIALS AND METHODS}

Ethics approval was provided by the University of Newcastle Human Research Ethics Committee before the study began (approval number H-2009-0270).

Four open-ended questions were used to understand how nuclear medicine personnel deal with patients belonging to groups that have been described as potentially difficult to question about pregnancy status $(2,4)$. Each question asked participants to "Briefly describe how you would question the patient in the following scenario": young teenager accompanied by a parent, unconscious or heavily sedated patient, patient with language or cultural barriers, and patient with a mental disability.

Content analysis (9) was used by the researchers to analyze the text and to identify several themes from the responses to each question. The analysis involved both quantitative and qualitative interpretation and analysis, allowing the data to be viewed in different yet complementary ways, with the outcome being the development of a range of distinct categories to describe the data. To eliminate potential bias by the primary researcher (a nuclear medicine technologist) and to ensure the validity of the theme categories, all responses were reviewed by the primary researcher and an independent researcher who had no working knowledge of nuclear medicine.

In the initial coding cycle, the researchers worked independently to examine the responses to each question with the intention of categorizing the responses into a variety of themes. One or more themes or subthemes could be contained within each participant response because more than one strategy might be used when questioning the patient. As there were over 200 responses for each question, the researchers began by reviewing the first 50 responses for each question. The researchers discussed the developing categories of description from the first coding cycle, and several major themes and subthemes were created and agreed on by both researchers. These themes were used to develop a source code book, which was used by both researchers to examine all remaining responses (Tables $1-4$ ).

\section{RESULTS}

There were 335 responses to the survey and $232(69 \%)$ responses to each of the open-ended questions regarding the 4 clinical scenarios. The participants who responded to the scenario questions were predominately female (69\%) and nuclear medicine technologists (93\%). There was no statistically significant difference between the distribution of $\operatorname{sex}(P=0.58)$ or profession $(P=0.12)$ for the main survey responses and the scenario responses. For all 4 questions, a 99\% agreement rate between the 2 independent reviewers validated the source code books.

Direct quotes have been selected from the responses to illustrate the findings. The quotes are coded with a number relating to the participant's identity (e.g., C83), and any correction of spelling or grammar has been underlined; for example: "Assess patient's attitude and demeanor."

\section{Young Teenager Accompanied by a Parent}

A total of 404 comments were grouped into 4 major themes with 16 subthemes (Table 1). The major themes were method of questioning (81\%), subjective assessment (5\%), determining risk of pregnancy (12\%), and pregnancy testing (2\%).

Method of Questioning (Theme 1). The most common strategy used to question a young teenager accompanied by a parent was to ask the girl away from the parent (49\%). This strategy could involve either asking the parent to leave

TABLE 1

Young Teenager Accompanied by Parent

\begin{tabular}{|c|c|c|}
\hline Major theme & Subtheme & $\%$ (normalized to major theme) \\
\hline \multirow[t]{6}{*}{ 1.1: Method of questioning (81\%) } & 1.11: Ask away from parent & 49 \\
\hline & 1.12: Ask with parent present & 15 \\
\hline & 1.13: Ask parent directly & $<1$ \\
\hline & 1.141: Same for all female patients, verbal question & 18 \\
\hline & 1.142: Same for all female patients, sign form & 7 \\
\hline & 1.15: Explain radiation risk & 10 \\
\hline \multirow[t]{3}{*}{ 1.2: Subjective assessment $(5 \%)$} & 1.21: Assess parent-child relationship & 25 \\
\hline & 1.22: Assess response & 65 \\
\hline & 1.23: Assess maturity level & 10 \\
\hline \multirow{4}{*}{ 1.3: Determining risk of pregnancy $(12 \%)$} & 1.31: Question regarding LMP & 38 \\
\hline & 1.32: Question regarding start of menstruation & 31 \\
\hline & 1.33: Question regarding if sexually active & 27 \\
\hline & 1.34: Question regarding $10-\mathrm{d}$ rule & 4 \\
\hline \multirow[t]{3}{*}{ 1.4: Pregnancy testing (2\%) } & 1.41: Pregnancy test & 44 \\
\hline & 1.42: Serum human chorionic gonadotropin & 22 \\
\hline & 1.43: Urine & 33 \\
\hline
\end{tabular}


TABLE 2

Unconscious or Heavily Sedated Patient

\begin{tabular}{|c|c|c|}
\hline Major theme & Subtheme & $\%$ (normalized to major theme) \\
\hline \multirow{4}{*}{$\begin{array}{l}\text { 2.1: Method of obtaining information on pregnancy } \\
\text { status }(71 \%)\end{array}$} & 2.11: Consult patient notes & 48 \\
\hline & 2.12: Consult nurse/caregiver/doctor & 27 \\
\hline & 2.13: Consult partner/relative & 18 \\
\hline & 2.14: Ask patient & 6 \\
\hline \multirow[t]{4}{*}{ 2.2: Pregnancy testing (14\%) } & 2.21: Pregnancy test & 23 \\
\hline & 2.22: Serum human chorionic gonadotropin & 62 \\
\hline & 2.23: Urine & 11 \\
\hline & 2.24: Ultrasound & 4 \\
\hline \multirow[t]{4}{*}{ 2.3: Unable to determine pregnancy status (13\%) } & 2.31: Do not perform scan & 27 \\
\hline & 2.32: Postpone scan & 42 \\
\hline & 2.33: Refer to nuclear medicine physician & 27 \\
\hline & 2.34: Reduce radiation dose & 3 \\
\hline 2.4: Never been in this situation $(2 \%)$ & & \\
\hline
\end{tabular}

the room or remain in the waiting room, taking the girl to another room (often under the guise of weighing her or taking her to a bathroom), or asking quietly so the parent could not hear.

"Ask the parent to wait outside while you set up and then ask the patient." (C70)

"We take the patient out of the room to get their weight and ask them while away from their parent." (C37)

"Try to do it quietly and without the parent hearing." (C65)

Questioning with the parent present was reported by $15 \%$, and 2 participants commented that they would ask the parent directly. Twenty-five percent commented that they question all female patients, regardless of age, in the same manner. Only $10 \%$ commented that they would explain the radiation risks from the procedure to the patient.

"Explain that radiation can have some risks to unborn foetus to child then mother, and explain that as a routine I ask all patients of childbearing age if they may be pregnant." (C193)

Subjective Assessment (Theme 2). Participants commented that in deciding whether to ask the patient about the possibility of pregnancy, they would make a subjective judgment to assess the parent-child relationship (25\%), the response from parent or child $(65 \%)$, or the maturity of the girl $(10 \%)$.

"Determine if they are comfortable talking in front of their parent." (C155)

“Assess patient's attitude and demeanor:" (C163)

Determining Risk of Pregnancy (Theme 3). When attempting to determine the risk of the patient being pregnant, 3 main questions were asked: date of last menstrual period (LMP) (38\%), whether the girl had commenced menstruating (31\%), and whether the girl was sexually active $(27 \%)$. Two participants $(4 \%)$ commented that they would use the 10 -d rule to assess the patient's risk of pregnancy.

"For younger teenagers we would ask first if they had gotten their period yet." (C90)

"Determine if sexually active and if so, use of contraceptive etc." (C79)

"Ask patient if menstruating. Ask date of LMP. If greater than 10 days determine if sexually active." (C118)

Pregnancy Testing (Theme 4). Comments regarding the use of pregnancy testing for teenagers made up only $2 \%$ of the comments. The type of pregnancy test used either was

TABLE 3

Patients with Language or Cultural Barrier

\begin{tabular}{|c|c|c|}
\hline Major theme & Subtheme & $\%$ (normalized to major theme) \\
\hline \multirow[t]{6}{*}{ 3.1: Method of questioning (91\%) } & 3.11: Use interpreter/translator & 67 \\
\hline & 3.12: Use visual aids & 27 \\
\hline & 3.13: Speak slowly & 1 \\
\hline & 3.14: If cultural barrier, use female staff & 2 \\
\hline & 3.15: Same for all female patients & 2 \\
\hline & 3.16: Do not ask & $<1$ \\
\hline \multirow[t]{3}{*}{ 3.2: Pregnancy testing (4\%) } & 3.21: Pregnancy test & 40 \\
\hline & 3.22: Serum human chorionic gonadotropin & 47 \\
\hline & 3.23: Urine & 13 \\
\hline \multirow{2}{*}{ 3.3: Unable to determine (4\%) } & 3.31: Consult with doctor & 38 \\
\hline & 3.32: Postpone scan if patient does not understand & 62 \\
\hline \multirow[t]{3}{*}{ 3.4: Determining risk of pregnancy $(1 \%)$} & 3.41: Question regarding LMP & 50 \\
\hline & 3.42: Question regarding contraception & 25 \\
\hline & 3.43: Question regarding sexual activity & 25 \\
\hline
\end{tabular}


TABLE 4

Patients with Mental Disability

\begin{tabular}{|c|c|c|}
\hline Major theme & Subtheme & $\%$ (normalized to major theme) \\
\hline \multirow[t]{7}{*}{ 4.1: Method of questioning (67\%) } & 4.11: Ask patient (slowly, simple language) & 26 \\
\hline & 4.12: Ask caregiver/relative & 50 \\
\hline & 4.13: Ask nurse/doctor & 11 \\
\hline & 4.14: Consult notes & 7 \\
\hline & 4.15: Use visual aids & 3 \\
\hline & 4.16: Same for all female patients & 2 \\
\hline & 4.17: Do not ask & 1 \\
\hline \multirow[t]{3}{*}{ 4.2: Determining risk of pregnancy $(7 \%)$} & 4.21: Question regarding LMP & 38 \\
\hline & 4.22: Question regarding contraception & 26 \\
\hline & 4.23: Question regarding if sexually active & 35 \\
\hline \multirow[t]{3}{*}{ 4.3: Pregnancy testing (17\%) } & 4.31: Pregnancy test & 41 \\
\hline & 4.32: Serum human chorionic gonadotropin & 38 \\
\hline & 4.33: Urine & 21 \\
\hline \multirow[t]{2}{*}{ 4.4: Subjective assessment (7\%) } & 4.41: Assume not sexually active & 3 \\
\hline & 4.42: Assess communication/understanding & 97 \\
\hline \multirow[t]{2}{*}{ 4.5: Unable to determine (1\%) } & 4.51: Do not perform scan & 60 \\
\hline & 4.52: Postpone scan & 40 \\
\hline
\end{tabular}

not specified (44\%) or was reported to be serum or blood testing $(22 \%)$ or urine testing $(33 \%)$.

\section{Unconscious or Heavily Sedated Patients}

A total of 467 comments were grouped into 4 major themes with 12 subthemes (Table 2). The major themes were method of obtaining information on pregnancy status $(71 \%)$, pregnancy testing (14\%), unable to determine status (13\%), and never been in this situation $(2 \%)$.

Method of Obtaining Information (Theme 1). For an unconscious or heavily sedated patient, rather than questioning the patient, the participants used a variety of methods to obtain information about pregnancy status. The most common method was to consult the patient notes (48\%). Participants commented that they would check the patient notes for information about recent pregnancy test results, contraceptive use, date of LMP, and length of stay in the hospital and to check for patient consent.

"Check medical records for LMP and length of stay in hospital and bHcg." (C7)

Other ways to obtain information were to consult with a medical professional (27\%), nurse, caregiver, or doctor, about the patient's pregnancy status or to ask the patient's partner or relative $(18 \%)$ if that person had accompanied the patient.

"Ask the nurse in charge of the patient the pregnancy status or last known menstrual period." (C55)

"Ask the partner if they are present." (C74)

Twenty participants $(6 \%)$ commented that they would attempt to question the patient.

"Would try to get an answer from heavily sedated patient but if I was not confident on answer I would not do test." (C41)

“Talk loudly with using actions.” (C85)

Pregnancy Testing (Theme 2). The comments of participants indicated that they were more likely to use pregnancy testing (14\%) for patients who were unconscious or heavily sedated, because the patient would be unable to answer their questions. Serum pregnancy tests were specified in 41 of 66 comments (62\%) regarding pregnancy testing.

"I wouldn't be comfortable going ahead in this situation unless there was a negative BHCG test available." (C122)

Unable to Determine (Theme 3). There were 62 comments $(13 \%)$ on what to do if the pregnancy status could not be determined. The most common response was to postpone the scan either until the patient was conscious and able to answer questions and provide consent or until pregnancy test results could be verified.

"Would not proceed-patient unable to consent for procedure.” (C13)

"Depending on how urgent the scan was and the level of suspicion, a blood test should be done or wait until they were conscious." (C152)

An equal number of participants (27\% each) stated that they would not proceed with the procedure or would defer to the nuclear medicine physician to make a decision as to whether to go ahead with the procedure.

Never Been in This Situation (Theme 4). There were 7 participants who commented that they had never faced this situation, usually because they worked in a private practice that did not cater to hospitalized patients.

\section{Patients with Language or Cultural Barriers}

A total of 371 comments were grouped into 4 major themes with 14 subthemes (Table 3). The major themes were method of questioning $(91 \%)$, pregnancy testing $(4 \%)$, unable to determine status (4\%), and determining risk of pregnancy $(1 \%)$.

Method of Questioning (Theme 1). The most common strategy for patients with a language barrier was to use an interpreter $(67 \%)$. This could be either a professional interpreter or a family member who spoke English. The use of visual aids was quite common (28\%). This category included referring to multilingual signage posted in the department, use of gestures or mime, drawing pregnant people, 
and the use of Google Translate to print out the questions in the relevant language.

"I would ask the patient if they were pregnant using either translation or we have a poster with this question asked in different languages." (C17)

“Mime being pregnant or holding a baby." (C41)

"Draw a picture/mime it out/interpreter/point to poster on wall.” (C84)

For patients with cultural barriers, the strategies suggested were to use female staff members to question the patient and to explain that the question needed to be answered because of the possible risk to the baby from radiation exposure.

"If it was cultural perhaps a female employee would do the questioning." (C227)

Pregnancy Testing (Theme 2). The major theme of pregnancy testing made up $4 \%$ of the total comments for this question. Serum pregnancy tests were most commonly recommended $(47 \%)$, whereas $40 \%$ did not specify the type of test in their comment. Urine pregnancy tests were suggested in 2 comments (13\%).

Unable to Determine Status (Theme 3). There were a small number of comments on what to do if pregnancy status could not be determined. In most cases $(62 \%)$, the procedure would be postponed or referred to either the nuclear medicine physician or the referring doctor.

"I would not do the test if I didn't have an informed answer." (C41)

Being unable to gain consent for the procedure if the patient could not understand was also a reason cited to postpone the procedure.

"If I am unable to ask the patient this question then it would also be assumed that I would not be able to gain consent for the examination anyway hence would not undertake the examination without the ability to communicate with them. The examination would need to be rebooked with an interpreter." (C130)

Determining Risk of Pregnancy (Theme 4). Only $1 \%$ of comments were on trying to determine the risk that the patient was pregnant by asking questions about LMP, contraceptive use, or sexual activity.

\section{Patients with Mental Disability}

A total of 469 comments were grouped into 5 major themes with 17 subthemes (Table 4). The major themes were method of questioning $(67 \%)$, subjective assessment $(7 \%)$, determining risk of pregnancy $(7 \%)$, unable to determine status $(1 \%)$ and pregnancy testing $(17 \%)$.

Method of Questioning (Theme 1). For patients with a mental disability, the most commonly used strategy to determine pregnancy status was to ask the caregiver or relative accompanying the patient $(50 \%)$.

"Talk to their caregiver if the patient could not understand." (C26)

Speaking slowly and using simple language to directly question the patient (26\%) was commonly used.

"Gesticulate, speak slowly and ask for understanding or ask them to repeat to you what they think you mean." (C68)
"Speak to the patient in simple and clear terms." (C158) Consulting with a medical professional responsible for the patient's care, such as a nurse or doctor $(11 \%)$, reviewing the patient's medical notes $(7 \%)$, and using visual aids (3\%) were some of the other strategies used to determine the pregnancy status for this type of patient.

Determining Risk of Pregnancy (Theme 2). Questions about the patient's LMP (38\%), sexual activity (35\%), or contraceptive use $(26 \%)$ are asked to determine the risk that the patient is pregnant. These types of questions are asked to the patient, the caregiver or relatives, and medical professionals.

"Ask them if they have a boyfriend or if they are trying to make a baby." (C73)

"Ask when was the last period/contraceptive use or ask caregiver.” (C120)

Pregnancy Testing (Theme 3). The use of pregnancy tests (17\%) to verify the pregnancy status of a mentally disabled patient is common, particularly if there is doubt whether the patient can understand verbal or written questions.

"If they are not able to understand or communicate then I will show her the poster of pregnant woman. If that doesn't work then wait for pregnancy test." (C50)

"Depends on level of disability. If unable to comprehend get a pregnancy test done.” (C58)

Subjective Assessment (Theme 4). There were several comments $(7 \%)$ indicating that participants make subjective assessments and assumptions about the level of understanding or disability of the patient or about the sexual activity of mentally disabled people. These assumptions are then used to decide on the method of questioning to be used.

"This would be dependent on the level of disability and degree of independence. If the incapacitation is profound, I may waive the questioning altogether." (C94)

"I would assume the patient is not sexually active if their mental disability is extensive." (C109)

\section{DISCUSSION}

This survey has revealed that nuclear medicine personnel in Australia and New Zealand use of a variety of strategies when determining the pregnancy status of a patient before diagnostic imaging procedures. Although the paper identifies the main strategies used for each clinical scenario, the participants' responses may include several different strategies that are used concurrently to attempt to gain a more accurate response from the patient and provide greater confidence in the decision-making process. For example, in the case of a patient with a mental disability, the patient and caregiver may be asked and a pregnancy test performed.

The most commonly used strategy described by the participants for each category was to attempt to question young teenagers without their parent present, consult the patient notes for unconscious or sedated patients, use an interpreter if the patient has any language or cultural barriers, and question the caregiver of mentally disabled patients.

Establishing the pregnancy status of a patient is one of the fundamental tasks performed before beginning any diagnostic 
nuclear medicine procedure using ionizing radiation to ensure the protection of any fetus. International and national radiation protection regulations (6-8) recommend that all women of child-bearing age be questioned on their pregnancy status, but the guidelines do not detail how the questioning should be performed. Our survey revealed wide variation in the methods used to question nuclear medicine patients in Australia and New Zealand (7). Variations in practice are a significant concern as they can lead to inconsistencies in the quality of health care and treatment received by individual patients. Clinical practice guidelines should be based on sound scientific evidence; however, in areas where there is a lack of evidence, practice patterns are often derived from the opinions or experience of the health professional (10).

Determining pregnancy status can be a sensitive issue and requires tact and discretion on the part of the health professional asking the questions. When attempting to determine pregnancy status before diagnostic imaging, the technologist tries to determine the risk of pregnancy by asking questions about the patient's menstrual cycle, sexual activity, and contraceptive use. The personal and intimate nature of this type of questioning can lead to embarrassment on the part of the patient, any accompanying persons, and even the technologist asking the questions. This is particularly so if the patient is unable to answer, such as for unconscious patients or patients with language barriers, and a relative or caregiver is asked the question instead. If the questioning is performed in an ad hoc manner, rather than using carefully developed protocols, a truthful answer or correct assessment may not be obtained, possibly resulting in irradiation of an unknown fetus. A study by James et al. (2) revealed that nuclear medicine technologists tend to assume that the patient is aware of her pregnancy status at the time of questioning, and a negative response may be accepted without any further questioning or testing.

There are conflicting reports in the literature concerning a woman's ability to self-assess her pregnancy status. Minnerop et al. (11) conducted a prospective, observational study on the ability of 377 adult women to exclude pregnancy without knowledge of a pregnancy test result. The researchers found that both patient history and physician suspicion could accurately exclude pregnancy. However, earlier studies by Strote and Chen (12) and Ramoska et al. (13) revealed that patient history alone was not adequate to exclude pregnancy, and both studies recommended that pregnancy testing be considered in emergency departments. In nuclear medicine, for which there is a real although relatively small risk that a fetus will receive ionizing radiation from a diagnostic maternal examination, it may be prudent to begin questioning patients about their history and to request a pregnancy test when there is any doubt.

Several patient groups have previously been identified as potentially difficult to question and obtain a truthful and accurate response (1-4). Young teenagers, especially when accompanied by a parent, are included in these groupings and may present a challenge for the nuclear medicine technologist. The technologist must decide first whether to question the patient about the possibility of pregnancy. This issue may be decided by the patient's age, whether she has commenced menstruation, or her apparent maturity and possibility of sexual activity. The required questioning is of a personal nature, and in some cases, subjective judgments are made by the technologist (2). Our survey revealed that if the teenager is accompanied by a parent or caregiver the most commonly used strategy is to question the teenager in a separate room or out of earshot of the parent, as it is believed that the teenager may not provide a truthful answer in front of her parent. This strategy raises ethical questions about the age of medical consent, confidentiality, and the legality of questioning minors without a parent or guardian present. The age of consent for medical procedures and treatment varies with the country and state. In general, in Australia, the United Kingdom, and the United States, consent from a parent or guardian is required for minors (children under the age of $18 \mathrm{y}$ ) for medical procedures or treatment. However, some states in Australia (New South Wales, South Australia, and Australian Capital Territory) have specific laws allowing younger children to consent for procedures. For example, in New South Wales, the Minors (Property and Contracts) Act (14) allows a child of $14 \mathrm{y}$ or older to consent to medical treatment. In states without consent laws, common law relating to the competency of a minor consenting to treatment may apply. This position was established by the English House of Lords decision in Gillick $v$ West Norfolk and Wisbech Area Health Authority (15). "Gillickcompetent" or "mature minor" children are deemed to be old enough or mature enough to make their own decisions and understand the issues and consequences regarding medical treatment. If children meet the Gillick definition, they are able to give consent and are entitled to the same confidentiality of medical information as an adult.

The survey responses regarding unconscious or heavily sedated patients, patients with language barriers, and patients with mental disabilities were often focused on the attempt to gain consent for a procedure as well as determine pregnancy status. Competency is considered a prerequisite for informed consent (16). However, there is a legal distinction between the requirement for capacity to consent and informed consent (17). The first focuses on the ability of a person to understand a proposed procedure or treatment, whereas the latter is concerned with the practitioner's duty to disclose information about the procedure. Informed consent is meaningful only when consenters are fully competent (meaning they possess the capacity to fully understand the situation and the ability to weigh potential outcomes and anticipate future consequences) and all relevant information regarding the procedure and any risks associated with the procedure have been conveyed to them (16).

\section{CONCLUSION}

This paper has discussed the methods used by nuclear medicine personnel in Australia and New Zealand to determine 
pregnancy status for 4 groups of patients considered to be potentially difficult to question. The most common strategies used were questioning young teenagers away from their parent, consulting patient notes for unconscious or sedated patients, using an interpreter for patients with language barriers, and questioning the caregiver of patients with mental disability. The survey revealed variations in current practice for determining pregnancy status before diagnostic nuclear medicine procedures that may lead to inconsistencies in the care and treatment of individuals from these groups. The development of best practice guidelines on how to question patients about their pregnancy status is recommended to ensure a consistent approach to questioning, which would optimize the accuracy of pregnancy assessment and reduce the possibility of fetal irradiation.

\section{DISCLOSURE}

No potential conflict of interest relevant to this article was reported.

\section{REFERENCES}

1. Applegate K. Pregnancy screening of adolescents and women before radiologic testing: does radiology need a national guideline? J Am Coll Radiol. 2007;4:533-536.

2. James DJ, Cardew P, Warren-Forward H. Determining the pregnancy status of patients before diagnostic nuclear medicine procedures: the Australian experience. J Nucl Med Technol. 2011;39:220-225.
3. Brent RL. Saving lives and changing family histories: appropriate counseling of pregnant women and men and women of reproductive age, concerning the risk of diagnostic radiation exposures during and before pregnancy. Am J Obstet Gynecol. 2009;200:4-24.

4. Cousins C. Medical radiation and pregnancy. Health Phys. 2008;95:551-553.

5. United Nations Scientific Committee on the Effects of Atomic Radiation. Sources and Effects of Ionizing Radiation: UNSCEAR 2008-Report to the General Assembly with Scientific Annexes. Vol II. New York, NY: United Nations; 2011:221-313.

6. Publication 84: pregnancy and medical radiation. Ann ICRP. 2000;30(1).

7. James DJ, Cardew P, Warren-Forward HM. Pregnancy screening strategies for diagnostic nuclear medicine: survey results from Australia and New Zealand. J Nucl Med Technol. 2013;41:216-222.

8. Safety Guide: Radiation Protection in Nuclear Medicine. Yallambie, Victoria, Australia: Australian Radiation Protection and Nuclear Safety Agency; 2008. Publication 14.2.

9. Dempsey SE, Warren-Forward HM. An analysis of the professional and academic interest of medical radiation science students. Radiography. 2011;17:145-151.

10. Murphy MK, Black NA, Lamping DL, et al. Consensus development methods, and their use in clinical guideline development. Health Technol Assess. 1998;2(3).

11. Minnerop MH, Garra G, Chohan J, Troxell R, Singer A. Patient history and physician suspicion accurately exclude pregnancy. Am J Emerg Med. 2011;29:212-215.

12. Strote J, Chen G. Patient self assessment of pregnancy status in the emergency department. Emerg Med J. 2006;23:554-557.

13. Ramoska EA, Sacchetti AD, Nepp M. Reliability of patient history in determining the possibility of pregnancy. Ann Emerg Med. 1989;18:48-50.

14. Minors (Property and Contracts) Act (NSW Government, 1970).

15. Gillick $v$ West Norfolk and Wisbech Area Health Authority, 1985 (U.K. House of Lords 7, 1986).

16. Koocher GP, Keith-Spiegel PC. Children, Ethics and the Law: Professional Issues and Cases. Lincoln, Nebraska: University of Nebraska Press; 1990.

17. Young People and Consent to Health Care. Sydney, Australia: NSW Law Reform Commission; 2008. Report 119. 